\title{
Integrating Innovative Neuro-educational Technologies (I-Net) into K-12 Science Classrooms
}

\author{
Ronald H. Stevens ${ }^{1}$, Trysha Galloway ${ }^{1}$, and Chris Berka ${ }^{2}$ \\ ${ }^{1}$ UCLA IMMEX Project, 5601 W. Slauson Ave. \#255, Culver City, CA 90230 \\ immex_ron@hotmail.com, \\ tryshag@gmail.com \\ ${ }^{2}$ Advanced Brain Monitoring, Inc, Carlsbad, CA 92008 \\ chris@b-alert.com
}

\begin{abstract}
With the U.S. facing a decline in science, math and engineering skills, there is a need for educators in these fields to team with engineers and cognitive scientists to pioneer novel approaches to science education. There is a strong need for the incorporation problem solving and emerging neuroscience technologies into mainstream classrooms, and for students and teachers to experience what it means at a very personal level, to engage in and struggle with solving difficult science problems. An innovating and engaging way of doing this is by making the problem solving process visible through the use of realtime electroencephalography cognitive metrics. There are educational, task, and measurement challenges that must be addressed to accomplish this goal. In this paper we detail some of these challenges, and possible solutions, to develop a framework for a new set of Interactive Neuro-Educational Technologies (I-Net).
\end{abstract}

Keywords: EEG, Problem solving, Skill Acquisition, Cognitive Workload.

\section{Introduction}

Science educators are increasingly being pressured to make their efforts more effective, efficient and relevant to the needs of today's workforce. Promoting "...an individual's capacities to use cognitive processes to confront and resolve real, crossdisciplinary situations where the solution path is not immediately obvious" is a worldwide educational priority (National Research Council, 2005, OECD, 2004). As tasks and problems become more complex, students face greater demands to hold and manipulate many forms of data in working memory. The burden placed on working memory (i.e., cognitive load) in these situations can have a significant impact on students' abilities to perform learning tasks and to benefit from them, because working memory capacity is limited (Baddeley, 2003, Sweller, 1989, 1994). Given the increasing consolidation of job functions within the workforce, the reality is that students will be assuming more responsibility for learning and decision making in the future, and will be increasingly confronted by data / information overload.

While students are individually struggling with increased mental demands, it is becoming more difficult for teachers to support their learning in meaningful cognitive 
ways. With limited training resources and staff size, rapid identification of students who are / are not developing problem solving skills has remained elusive (National Research Council, 2004). Part of the challenge is cognitive. Strategic problem solving is complex with skill level development being influenced by the balance of the task and the experience and knowledge of the student. Another challenge is observational. Assessment of problem solving requires real-world tasks that are not immediately resolvable and require movement among structural, visual and auditory representations. Lastly, there are challenges of how rapidly valid inferences of the cognitive state of a student can be made and reported from the performance data.

Application of neurophysiologic solutions, especially electroencephalography (EEG) to education offers cutting-edge approaches to these educational challenges. First, with a temporal resolution of seconds or milliseconds the goal of real-time feedback could optimize engagement and cognitive workload during learning. As EEG data collection occurs without interfering with normal task-driven cognition, it can provide an assessment of cognitive load that is not confounded by strong measurement effects. Further on the horizon would be the use of brain-computer interfaces to drive learning in ways that remain in the domain of science fiction.

With today's technologies, classroom-wide application of EEG during educational tasks could individualize the learning experience in specific ways. For example: Existing monitoring devices could detect situations where the student is simply not ready to learn. This could arise through sleep deprivation, a common occurrence among pre-teens and teens (Meijer et al, 2000), or excessive stress / poor stress management. Under these situations classroom instruction may fail, and errors in the learning process will increase

There may also be situations where the task exceeds the individual's immediate ability to mentally model the problem due to the difficulty of the task with respect to content / language and the student cannot be adequately engaged in the learning process although they are motivated to do so. This would be evidenced either by disengagement or 'thrashing', (acquiring excessive data while not engaged or while not processing it), conditions that could lead to poor decision making. Finally there are conditions where distractions and constraints in the environment combine to make a task that a student would normally easily learn, difficult. Such constraints include time pressure, noise, distraction and/or peer pressure. Were these situations quickly recognized and corrected then learning could become accelerated and more personalized.

Throughout education, there is a wide gap in translating the findings of cognitive neuroscientists into everyday educational practice (Goswami, 2006). The challenges are multi-factorial involving issues of diffusion of innovations, disconnects between scientists and educators, and issues of technology and turf. In this paper we draw from multiple perspectives of technology and education to explore possible pathways for taking I-NET into the messy world of real tasks and classroom environments. We first outline the major challenges along the way and close with a near vision of what these approaches can contribute to improving the learning of students.

Our starting framework for situating I-NET tools in education includes the:

- Parameters for measures and devices

- Approaches for situating neurophysiologic measures in education, 
- Specifications for the design and delivery of meaningful tasks, and guidelines regarding the capture of cognitive measurements and reporting of findings.

In doing so, we draw from programmatic lessons of the initial DARPA Augmented Cognition Program which emphasized the need for these technologies to "extract meaningful cognitive measures for identified periods of interest", as well as to "situate themselves within environments where they demonstrate high visibility effects that satisfy near as well as long-term needs" ("The End of the Beginning", Washington DC, 1/26/07).

\section{EEG as a Neurophysiologic Method of Choice}

For routine use in classroom settings I-NET instrumentation should be non-obtrusive for the students and the classroom environment, easy for students to apply, applicable to multiple task formats, inexpensive, and easy to collect meaningful data from. Realtime EEG monitoring satisfies many of these requirements. Technically, EEG-based cognitive load measurements offer the advantage of extremely high temporal resolution. Electrophysiological data is collected at a grain-size of tenths of a second, in contrast to the tens or hundreds of seconds required for traditional measures. This opens the possibility for effective monitoring of workload fluctuations during very rapid decision-making processes that are unobservable using traditional methods. Although there is a large and growing literature on the EEG correlates of attention, memory, and perception (Fabiani, 2001) there is a relative dearth of EEG investigations of the process skill acquisition and learning in classrooms (Smith,1999). Recording and analysis of EEG has traditionally been confined to laboratory settings and training to criterion tasks due to the technical obstacles of recording high quality data and the computational demands of real-time analysis.

Advances in electronics and data processing set the stage for ambulatory EEG applications. A recently developed wireless EEG sensor headset facilitates easy acquisition of high quality EEG combining battery-powered hardware with a sensor placement system to provide a lightweight, easy-to-apply method to acquire and analyze six channels of high-quality EEG. These headsets have been developed by Advanced Brain Monitoring, Inc. and are useful for in-the-field studies where the setup and acquisition of high quality recordings can be obtained by the user without technical assistance. Such units capture EEG data and provide real-time reports of (a) working cognitive load (EEG-WL), (b) distraction (EEG-DT) and (c) engagement (EEG-E). Quantification of the EEG in real time, referred to as the B-Alert system, is achieved using signal analysis techniques to identify and decontaminate eye blinks, and identify and reject data points contaminated with electromyography, amplifier saturation, and/or excursions due to movement artifacts (Berka et al., 2004). The EEG sensor headset requires no scalp preparation and provides a comfortable and secure sensor-scalp interface for 12 to 24 hours of continuous use.

Recent validation studies that included tests of forward and backward digit span, 3choice vigilance, standard image recognition, verbal paired associate recognition, etc suggest that EEG-E reflects information-gathering, visual scanning and sustained attention. EEG-WL increases with increasing working memory load and during 
problem-solving, integration of information, analytical reasoning and may be more reflective of executive functions. Inspection of EEG on a second-by-second timescale has revealed associations between workload and engagement levels when aligned with specific task events providing preliminary evidence that second-by-second classifications reflect parameters of task performance (Berka et al., 2004). These associations have been demonstrated with adults in tasks such as driving and weapons training simulation. We have extended this approach to investigate the role of cognitive effort (workload, distraction, and engagement) in high school students' math and science learning.

\section{Challenges in the Educational System}

An innovation has to be important to the teachers and administrators of a school system if it is going to be used; if it is just based on pure research protocols it probably won't be (Rogers, 1995). A proposed educational innovation should align with existing educational activities and practice; or be used to extend or expand the existing curriculum. Our interactions with educators have approached these challenges from both perspectives.

Maximizing the benefits of EEG technologies, involves developing an understanding of what students and teachers are looking at. Developing a firm grounding in the basic neuroscience and in the clinical neurology of EEG is needed. One approach for expanding the curriculum is through the development of a Brain, Mind and Learning Tool-kit for high school science teachers that features systems and software for acquisition, analysis, and interpretation of the EEG. Introducing brain monitoring into the classroom creates the opportunity for content-rich training in neuroscience, psychology, and a hands-on experience for students and teacher to observe their brain activity in real-time and understand how different states of mind are reflected in the electric fields of the brain. Study of EEG can contribute to a better understanding of the physics of energy, electricity, and waves, as well as the chemistry of biosynthetic pathways and signaling cascades. These workshop / hands-on activities build awareness and understanding of the science and the goals of the project including broad issues of investigation and experimentation.

An alternative method of providing value to the educational system is to incorporate tools that complement existing curricular activities and extend them by providing fast, accurate and more detailed information to support student learning. Using second-by-second EEG and detailed descriptions of cognitive events during the performance of complex tasks provides models for mediating the tasks, and/or the stress on an individual when workload exceeds an optimal threshold. By studying the dynamics of cognitive patterns within different classroom environments new insights are gained about complex problem solving, collaborative learning tasks and hands-on and minds-on science activities.

Example questions that could be approached include:

- What are the ranges and dynamics of cognitive workload and engagement with students demonstrating different levels of problem solving proficiency? Are these features stable, or do they change with experience, various domains and the acquisition of skills? 
- What would be the likely classroom impacts if a teacher were able to monitor a graphic display tracking the engagement, distraction and workload of an entire class in real time?

- How do cognitive workload and engagement levels differ for students performing a simulation vs. observing a simulation?

\section{IMMEX $^{\mathrm{TM}}$ Scientific Problem Solving Tasks}

From this spectrum of educational possibilities we have aligned our research activities with complex problem solving tasks that are being used extensively throughout school districts across this country and other nations such as China. This problem solving simulation system, termed IMMEX (Interactive Multi-Media Exercises) contains dozens of standards-based science simulations suitable for middle and high school classrooms. Over 600,000 student performances have been obtained on these simulations leading to the construction of probabilistic models of performance and skill acquisition (Stevens et al, 1999, Stevens \& Casillas, 2006). IMMEX simulations are also cognitively complex and most student activities while engaged in problem solving can be mapped to specific elements of cognition (Chung et al, 2002). A final advantage of IMMEX is that simulations exist in many science domains and across grade levels; students will often perform over 100 simulations each year in domains as diverse as math, chemistry, earth science and health. Aligning I-NET tools into such an activity could provide high project visibility throughout the school district.

Deriving EEG measures during open-ended problem solving is not easy. The development of problem solving skills is a gradual process and not all novices solve problems in the same way, nor do they follow the same path toward understanding at the same pace. Given the diversity of the student population it can be difficult to assess what their relative levels of competence are when performing a task, making it difficult to accurately relate EEG measures to other measures of task skill. In realworld educational settings there is not anything approaching the control over the relevant experimental parameters like others studying the sensory and motor systems. There one knows exactly when light hits the retina; however, how does one determine when a memory has occurred on a millisecond timescale?

Complex problem solving activities can critically depend upon the student's motivational and emotional states, creating additional complexity and variability when compared with automatic behavioral responses, or even voluntary motor responses. The memory traces of learning are not necessarily formed with any reproducible temporal offset relative to the time the student demonstrates the memory behaviorally, and this temporal ambiguity makes the job of ascribing meaning to a particular firing correlate especially difficult as we are less sure of when to look, or indeed what a neural correlate of problem solving might look like.

Our approach decomposes problem solving into behaviorally relevant segments and is much like an exercise in situation awareness where data is continuously acquired and consolidated to provide a 'big picture' understanding of the problem. Components of such awareness include perception of the elements in the environment, comprehension of the elements, and prediction of future interaction and 
events. The analogous components in problem solving are framing, navigation and investigation, and closure.

Framing. The perception analog in IMMEX tasks is a framing event. Text or multimedia descriptions are presented describing a situation, and posing one or more challenges. For example, in the middle school problem set Phyto Phyasco the prologue describes a newspaper clip indicating that a local farmer's potato crop is mysteriously dying; the challenge for the student becomes to determine if this is due to pests, lack of nutrients, infection, etc.

Successful framing is critical for solving problems and experts often spend a disproportionate amount of time on framing. Similarly, in situation awareness, the majority of errors are based on errors in perception (Endsley, 1999). If the student reads the prologue too quickly or does not take notes, or does not sufficiently understand the terms then the framing process could fail. Occasionally students become so involved in the details of the problem solving itself that they forget what the question being asked is and need to reframe the problem. Currently it is very difficult to know if the student has correctly framed the problem without extensive verbal questions and answers. EEG can help illuminate the framing process by determining if the student is engaged or overwhelmed by the task being proposed. We have also observed that as the student gains problem solving experience on the tasks the level of workload needed for effective framing begins to decrease (Stevens et al, 2007).

Navigation and Integration. Once the problem is framed the next important step is the identification of the most relevant resources available to solve the problem. All IMMEX tasks contain a hierarchy of resources that students navigate while gathering information. Figure 1 shows the problem space for Phyto Phyasco which consists of 5 major categories, each containing multiple sub categories of information.

The top menus are mainly used for problem space navigation. The sub menu items to the left are more decision making points as there is a cost or risk associated with each. In the problem set Phyto Phyasco there are 38 choices. What results is a dynamic iterative process of data collection and integration from which the solution to the problem is derived.

This is a complex process as few students solve IMMEX problems using the same sequence of actions, and currently it is not possible to determine from the framing, navigation, and decision events alone whether the student will solve the problem. Some students may have the relevant information in hand but do not understand the significance of it in the context of the problem. Other students through either bias or poor framing will never acquire the most useful information. Still others will access all the available information in a non-discriminating way without a clear hypothesis. Probabilistic descriptions of navigation and integration can provide a proxy for student understanding, but the models often lack the precision and temporal updating needed for a global metric of student's situation awareness (Stevens \& Casillas, 2006).

During efficient navigation and investigation, students often demonstrated a cycling of the B-Alert cognitive indexes characterized by relatively high workload and low engagement which then switched to lower workload and higher engagement. These cycle switches were often, but not always associated with selection of new data items. 


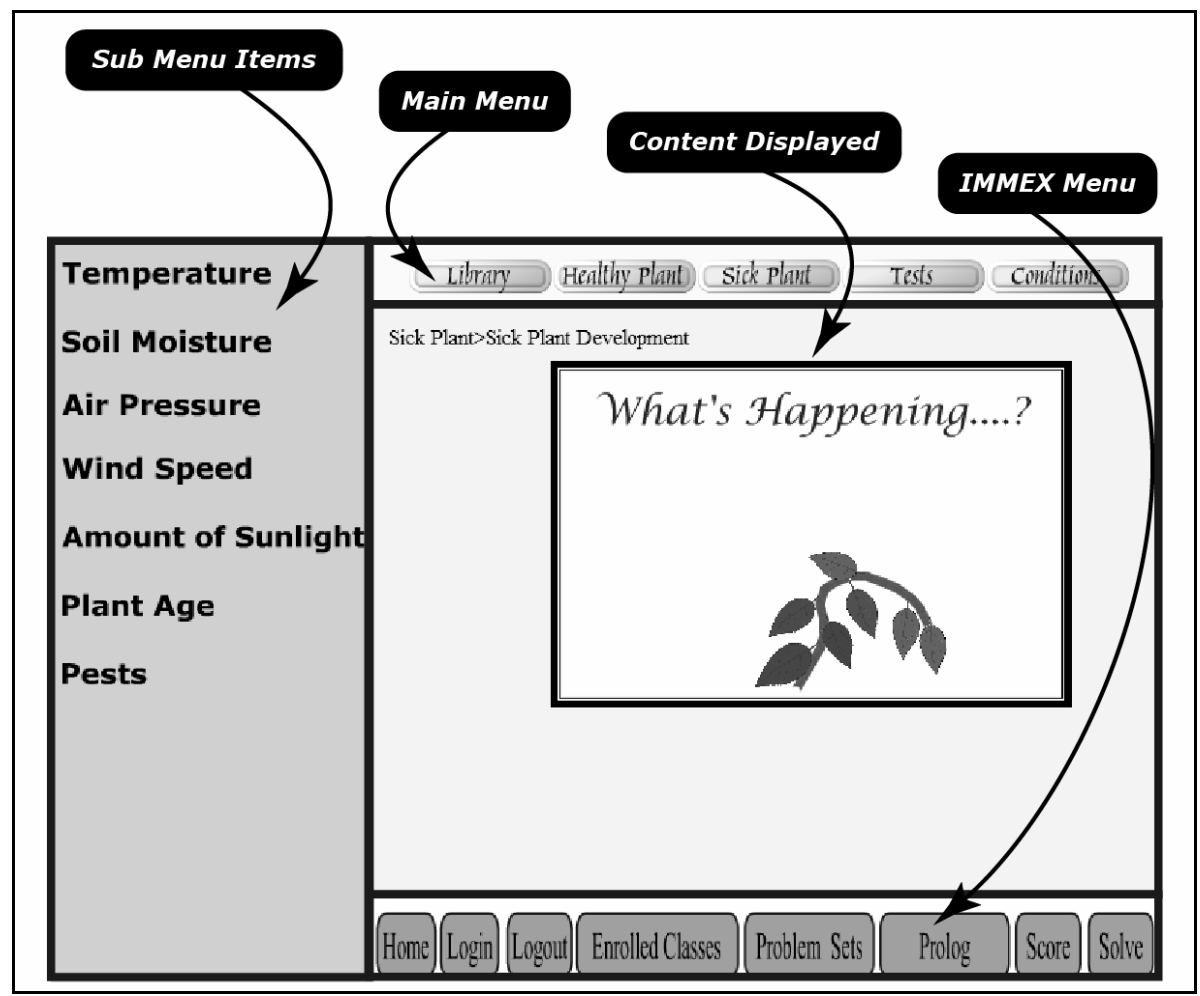

Fig. 1. Sample IMMEX ${ }^{\mathrm{TM}}$ simulation. In the Phyto Phyasco simulation, potato plants are dying and the student must identify the cause by examining weather conditions, nutrients, etc. Students navigate throughout the problem space using the Main Menu and select data to make decisions using the Sub Menu Items.

Multiple EEG correlates exist that are useful for understanding the navigation and integration events. On complex tasks, humans seem to use prior information to generate predictive codes for anticipated future events and continually compare incoming data with these codes (Summerfield et al, 2006). Perception-related evoked response potentials (ERP) similar to the auditory mismatch negativity (MMN) (Naatanen, 2001), may be observed when data is displayed that is not consistent with the current hypothesis. The absence of such signals could also be important if a deviant source of data was not recognized when presented.

It is also likely that there will be differences in engagement and workload when specific items of data are seen for the first time vs. subsequent times. The first time a piece of data is examined the student must categorize both the representation of the data, as well as associate the importance of the data to the context of the problem. Once the representation of the data is understood then the subsequent data viewing should only relate to the problem context and workload and engagement measures would be expected to be shorter or lower. At the level of ERP, a decrease in the latency of the P300 wave may be seen once the data representation is learned (Luck, 1998). 
Closure. Navigation and investigation is an open-ended process where the student can continue until s/he is satisfied that a solution has been obtained and closes the problem. Here the students first decide to solve the problem, an irreversible step in the sense that once this decision is made then a solution must be entered. Then the students select a solution from a list, and confirm the selection. There is no global cognitive metric for knowing the likely problem solving outcome; however, having such a metric would be important for learning as student's success and approaches to problem solving can persist for months after initial learning.

The steps leading to closure are highly dynamic and rich in uncertainty and errors due to premature closure (errors perceived as errors), as well as errors due to data limitations (errors about which there was uncertainty) can be observed which may evoke error-related negativity ERP (Scheffers \& Coles, 2000). On IMMEX tasks, the more productive closure sequences are preceded by the high workload, rather than high engagement by B-Alert measures.

\section{The Near Future}

Figure 2 portrays an interface to the I-NET workbench which will link student's neurophysiologic metrics, problem solving actions, and machine learning models of performance and progress. It shows the screen of a teacher who is monitoring six of her students for Engagement, Distraction and Workload using the B-Alert sensor software. Each bar in the histograms represents averaged metrics at 1-second epochs.

Panels A, C and to a lesser extend F most closely represents students who are productively engaged in problem solving; workload levels are moderate and the levels are alternating with cycles of high engagement. Many of these cycles are associated with navigation and interpretation events. Panel B illustrates a student who may be experiencing difficulties and may not be prepared to learn. The workload and engagement levels are low and distraction is consistently high. Under these conditions the student should be asked to terminate the task as productive learning is not likely to occur.

The student in Panel D has just encountered a segment of the simulation that induced 10-15 seconds of distraction (middle row) and decreased workload and engagement. By clicking on those EEG segments the teacher retrieved a view of the data that the student was looking at, which in this case is an animation of a plant growing. She could then make a note to discuss with the student to determine the source of the distraction and / or to see if others in the class have struggled with the same or similar issues.

Panel E shows a student who, while not distracted, appears to be working at beyond optimal capacity with workload levels consistently near 100\%. The teacher then retrieved the artificial neural network performance assessment models for this student (Stevens et al, 2004, Stevens \& Casillas, 2006) which shows that this student does not seem to be developing efficient strategies on his own. Utilizing this information, the instructor may suggest the adjustment of the difficulty of the task for this student and continue monitoring to see if more acceptable (in a learning sense) levels of workload and engagement result. 


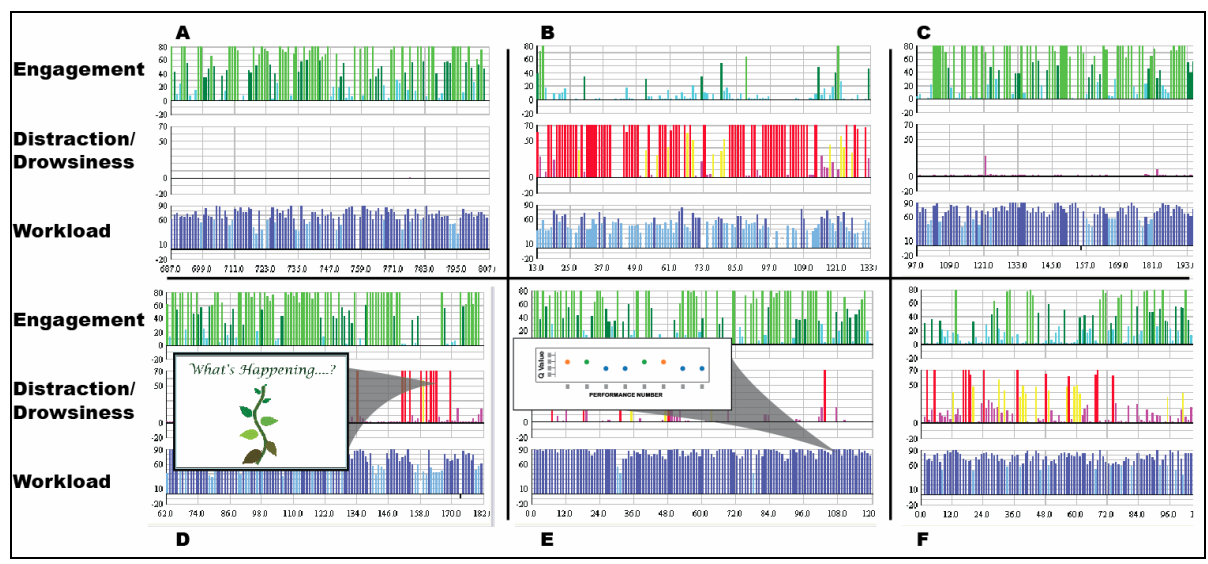

Fig. 2. Prototype I-NET interface for linking EEG measures of workload, distraction and engagement with details of the tasks and probabilistic models of problem solving performance and progress. While the interface itself is not yet functional, the tasks, EEG readings and machine learning model reports are actual student performance data.

Acknowledgements. This project has been funded by several grants from the National Science Foundation, (DUE- 01236050, REC-02131995, NSF-ROLE 0528840, HRD-0429156, DUE-0512526) whose support is gratefully acknowledged.

\section{References}

1. Baddeley, A.: Working Memory: Looking Back and Looking Forward. Nature Reviews I Neuroscience 4, 829-839 (2003)

2. Berka, C., et al.: Real-time analysis of EEG indexes of alertness, cognition and memory acquired with a wireless EEG headset. International Journal of Human-Computer Interaction 17(2), 151-170 (2004)

3. Berka, C., Levendowski, D.J., Cvetinovic, M., Petrovic, M.M., Davis, G.F., Lumicao, M.N., Popovic, M.V., Zivkovic, V.T., Olmstead, R.E.: Real-Time Analysis of EEG Indices of Alertness, Cognition and Memory Acquired with a Wireless EEG Headset. International Journal of Human-Computer Interaction 17(2), 151-170 (2004)

4. Chung, G.K.W.K, deVries, L.F., Cheak, A.M., Stevens, R.H., Bewley, W.L.: Cognitive Process Validation of an Online Problem Solving Assessment. Computers and Human Behavior 18, 669 (2002)

5. Endsley, M.R.: Situation awareness in aviation systems. In: Garland, D.J., Wise, J.A. (eds.) Handbook of aviation human factors. Human factors in transportation, pp. 257-276. Lawrence Erlbaum Associates, Mahwah (1999)

6. Fabiani, M., Gratton, G., Coles, M.G.: Event-related brain potentials. In: Caciooppo, J.T., Tassinary, L.G., Berntson, G.G. (eds.) Handbook of Psychophysiology, pp. 53-84. Cambridge University Press, Cambridge, England (2000)

7. Goswami, U.: Neuroscience and education: from research to practice? Nature Reviews Neuroscience 7, 2-7 (2006)

8. Luck, S.: Sources of dual-task interference: Evidence from human electroencephalography. Physiological Science 9, 223-227 (1998) 
9. Meijer, A.M., Habekothe, H.T., van Den Wittenboer, G.: Time in bed, quality of sleep and school functioning of children. J. Sleep Res. 9, 145-153 (2000)

10. Naatanen, R.: The perception of speech sounds by the human brain as reflected by the mismatch negativity (MMN) and its magnetic equivalent (MMNm). Psychophysiology 38, 1-21 (2001)

11. National Research Council, Knowing what students know: The science and design of educational assessment. Committee on the Foundations of Assessment. In: Pelligrino,J., Chudowsky, N., Glaser, R. (eds.) Board on Testing and Assessment, Center for Education. National Academy Press, Washington. DC (2001)

12. National Research Council, Rising Above the Gathering Storm: Energizing and Employing America for a Brighter Economic Future (2005)

13. Organization for Economic Cooperation and Development, Problem Solving for Tomorrow's World, First Measures of Cross-curricular competencies from PIS 2004. p. 26 (2004)

14. Rogers, E.M.: Diffusion of Innovations, 4th edn. Free Press, New York (1995)

15. Scheffers, M.K., Coles, M.: Performance monitoring in a confusing world: Error related brain activity, judgments of response accuracy and types of errors. Journal of Experimental Psychology: Human Perception and Performance 26, 141-151 (2000)

16. Stevens, R., Casillas, A.: Artificial Neural Networks. In: Mislevy, R.E., Williamson, D.M., Bejar, I. (eds.) Automated Scoring, pp. 259-312. Lawrence Erlbaum, Mahwah (2006)

17. Stevens, R.H., Ikeda, J., Casillas, A., Palacio-Cayetano, J., Clyman, S.: Artificial neural network-based performance assessments. Computers in Human Behavior 15, 295-314 (1999)

18. Summerfield, C., Egner, T., Greene, M., Koechlin, E., Mangels, J., Hirsch, J.: Predictive codes for forthcoming perception in the frontal cortex. Science 314, 1311-1315 (2006)

19. Sweller, J.: Cognitive technology: Some procedures for facilitating learning and problem solving in mathematics and science. Journal of Cognitive Psychology 81(4), 457-466 (1989)

20. Sweller, J.: Cognitive load theory, learning difficulty, and instructional design. Learning \& Instruction 4(4), 295-312 (1994) 